\title{
MESHLESS SOLUTION OF INCOMPRESSIBLE FLOW OVER BACKWARD-FACING STEP
}

\author{
Juraj MUŽÍKK ${ }^{1, *}$ \\ ${ }^{1}$ Department of Geotechnics, Faculty of Civil Engineering, University of Žilina, Univerzitná 1, 01026 \\ Žilina, Slovakia. \\ * corresponding author: muzik@fstav.uniza.sk.
}

\begin{abstract}
Article presents the use of the meshless method for numerical simulation of incompressible fluid flow. The article presents the implementation of the meshless local Petrov-Galerkin method (MLPG), with Navier-Stokes equation formulated using the local weighted residual principle. The trial function construction process is the most important part of the meshless method implementation. In this article the radial basis functions (RBF) are used for the process of the trial functions construction.
\end{abstract}

\section{Keywords:}

Meshless analysis; Meshless Petrov-Galerkin method;

Navier-Stokes equations;

Numerical simulation.

\section{Introduction}

One of the most important problems in the field of computational fluid dynamics (CFD) is solution of Navier-Stokes (NS) equations, which describes the incompressible fluid flow. In the many numerical simulation cases the NS equations are used in the alternative form such as velocity-vorticity or stream-vorticity form. In the case of mentioned NS equations forms the numerical solution is simpler than using the primitive variable (velocity, pressure) form. The primitive variable incompressible NS equations represent so-called mixed formulation without any explicit equation for pressure field. For mixed formulation numerical problems, the Babuska-Brezzi (BB) condition should be satisfied in order to maintain numerical stability of the simulation. The BB condition is often satisfied using different order of trial functions for velocity and pressure field. Using such approach is difficult with meshless methods, so other methods are used to overcome mixed formulation difficulties. The other kind of problem in the numerical solution of incompressible NS equations is the treatment of nonlinear convection term, which causes the velocity solution oscillations. The first use of meshless method for the solution of the incompressible NS equations formulated using primitive variables was presented by Lin and Atluri (2001). In their article they satisfied the BB condition using the continuity equation modified by perturbation term. For successful treatment of the convection terms in the momentum equation they presented two upwind schemes based on the test function skew and support domain translation. But the problem with determination of the perturbation parameter in the case of high Reynolds number still persist and no suitable rigorous way was presented. In the present work the meshless local Petrov-Galerkin (MLPG) method is used, in contrast with Lin and Atluri (2001) the time dependent momentum equation forms is used. The velocity-pressure decoupling is achieved using fractional step algorithm (Sataprahma and Luadsonga, 2013).

\section{Governing equations and fractional step algorithm}

The unsteady incompressible viscous fluid flow is described by Navier-Stokes equations with the continuity equation in the convection term (Sataprahma and Luadsonga, 2013). These equations can be described as follows

$v \frac{g^{2} u_{i}}{g x_{j} g x_{j}}-\frac{1}{p} \frac{g p}{B x_{i}}-\frac{a}{B x_{j}}\left(u_{j} u_{i}\right)-\frac{\partial u_{i}}{g t}=f$

$\frac{2 u_{\bar{L}}}{2 x_{i}}=0$ 
$u_{i}$ is the velocity, $p$ is the pressure, $f_{i}$ is the component of body force, $\rho$ is a liquid density and $v$ is the kinematic viscosity. The equation (1) is the momentum equation and (2) is the continuity equation. The governing equations $(1,2)$ are processed using fractional-step algorithm (Kovarik et al., 2014). The forward difference is used to approximate the time derivative of the velocity field and the equation (2) takes the following form

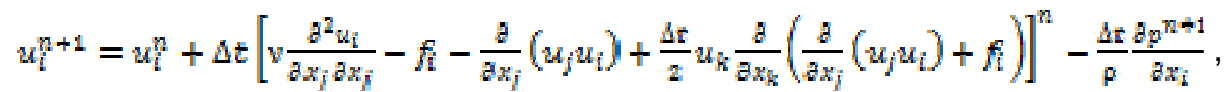

time step is marked by upper indices $n$ and $n+1$. In the formulation of momentum equation (3) the diffusion and convection terms are treated explicitly and the pressure term is treated implicitly. The next step in the fractional step algorithm is the simplification of the momentum equation by dropping the pressure term (Kovarik et al., 2014) to obtain simplified form of the momentum equation (4).

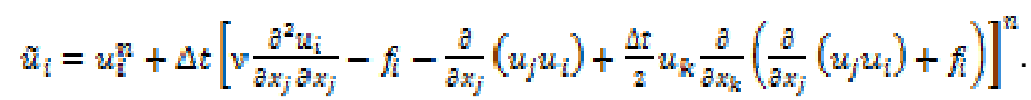

The velocity components $\tilde{u}_{i}$ obtained by the equation (4) are called intermediate velocities and these values do not satisfy the continuity equation, e.g. the intermediate velocity field is not divergence free.

By the comparison of equations (3) and (4) we get following equation

$u_{i}^{n+1}=u_{i}-\frac{s t}{p} \frac{\partial p^{n+1}}{\partial x_{i}}$.

The velocities $u_{i}^{n+1}$ must satisfy the continuity equation. So in the next step the intermediate velocities are corrected using the projection method described by following Poisson equation

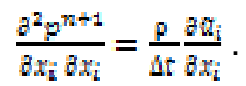

Equation (6) is the pressure equation with non-zero source term (Kovarik et al., 2012). The MLPG method is used to discretize and solve the pressure field over domain of interest with the following boundary conditions $p^{n} \mid \Gamma_{u}=\bar{p}^{n}$ and $\partial p^{n} / \partial n=\bar{q}^{n}$.

\section{The MLPG Method and the Local Weak Formulation}

The meshless local Petrov-Galerkin method (MLPG) is truly meshless method which requires no internal cells or elements connected to global mesh, for either interpolation or integration purposes. In MLPG the solution domain is formed by a set of scattered nodes (Kovarik, 2011). 


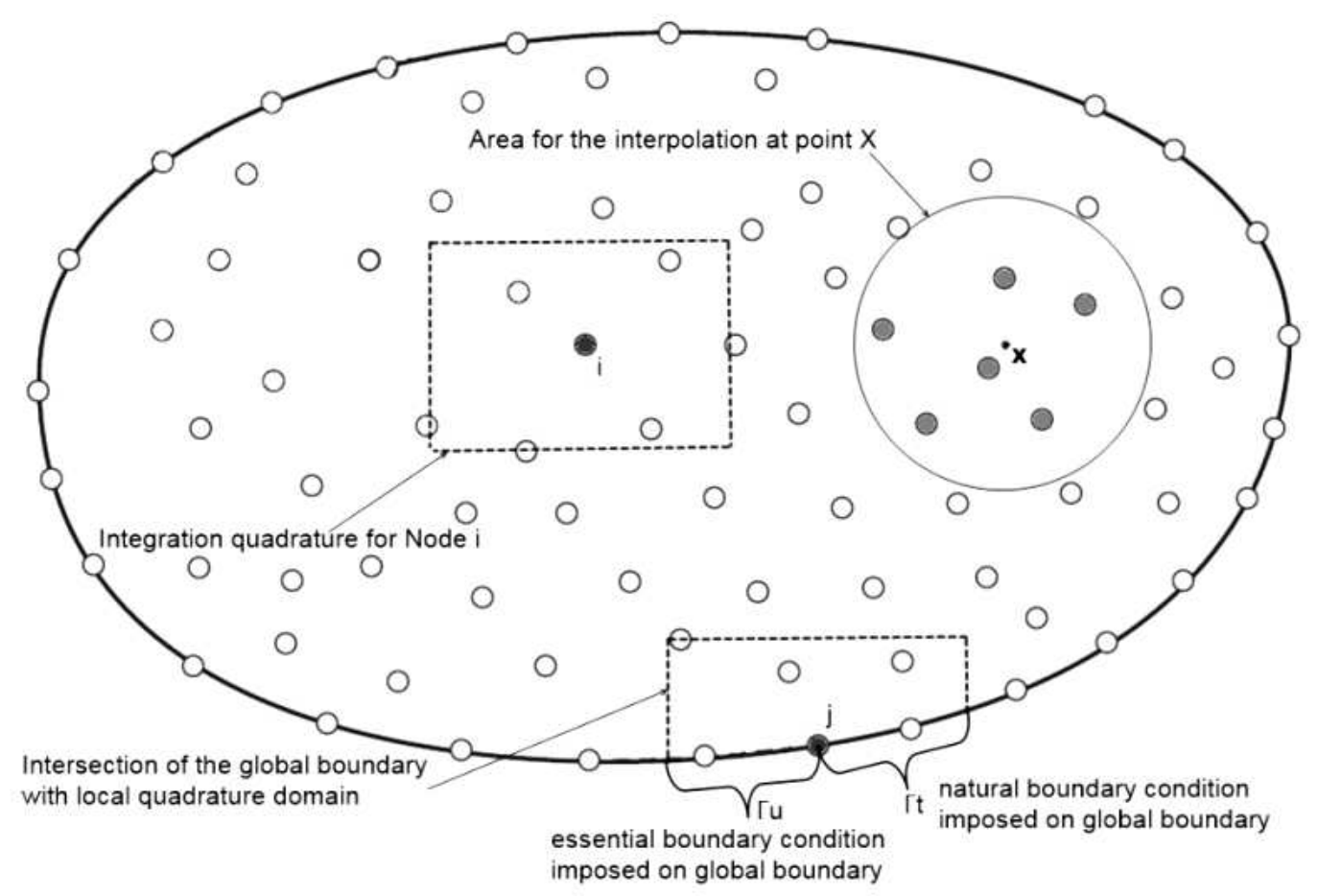

Fig. 1: Local quadrature domain and support domain for interpolation.

The linear system of discrete equations describing the computational problem is obtained using the weighted residual form of the governing equation integrated over local mesh of cells (see. Fig.1). The shape of the integration cell can be arbitrary in theory, but for simplicity of the implementation the simple regular shapes are used, such as rectangles for 2D case (Izvoltova and Villim, 2012).

The pressure equation (6) in the integral (weak) form defined over local sub-domain $\Omega_{s}$ can be written as follows

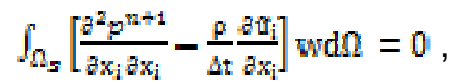

where $p$ is pressure, $w$ represents the weight (test) function that has following form

$w(\eta)=\left\{\begin{array}{ll}1-6 \eta_{i}^{2}+8 \eta_{i}^{2}-3 \eta_{i}^{4} & \eta_{i} \leq 1 \\ 0 & \gamma_{i}>1\end{array} ; \quad \eta=\frac{\left\|x-x_{i}\right\|}{d_{s}}\right.$,

where $d_{s}$ represents the local integration cell dimensions. From the definition of the weight function (8) one can see it has value of zero on its boundary. The choice of this form of test function is motivated by its ability to vanish on the boundary of local quadrature domain. Integrating of the (7) by parts the following form is obtained

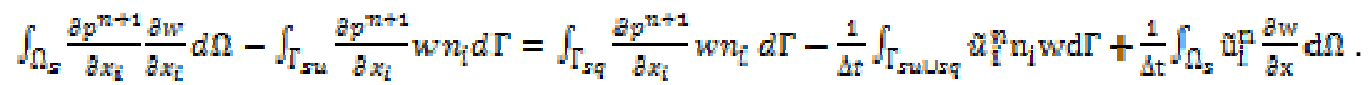

The unknown values of the pressure $p$ at the computational nodes are constants and can be moved out of the integration operation in the (9). The one can set discrete system of linear equations using (9), where global characteristic matrix is expressed as follows

$K_{m}=\int_{\Omega m} \frac{B \phi_{n}}{B x_{i}} \frac{B w_{m}}{B x_{i}} d \Omega-\int_{\Gamma \mathrm{m}} \frac{B \phi_{\mathrm{m}_{\mathrm{m}}}}{B \mathrm{x}_{\mathrm{i}}} \mathrm{W}_{\mathrm{m}} \mathrm{n}_{\mathrm{i}} \mathrm{d} \Gamma$

and the definition of right-hand side "forcing" vector is following

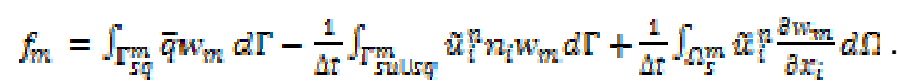


The term $\varphi$ in equation (10) represents the trial function, in this case the Multi-Quadrics Radial Basis function (MQ-RBF); details can be found in Kovarik (2011). The weak form of the equations (4) and (5) can be written as follows, assuming for simplicity $f_{i}=0$

$\int_{\Omega_{s}} w \tilde{u}_{i} d \Omega=\int_{\Omega_{s}} w u_{i}^{n} d \Omega+\Delta t \int_{\Omega_{s}} w\left[v \frac{g^{2} u_{i}}{\partial x_{j} g x_{j}}-\frac{\partial}{\partial x_{j}}\left(u_{j} u_{i}\right)+\frac{\Delta t}{2} u_{k} \frac{\partial}{\partial x_{k}}\left(\frac{g}{\partial x_{j}}\left(u_{j} u_{i}\right)\right]^{n} d \Omega\right.$,

$\int_{\Omega_{s}} w u_{i}^{n+1} d \Omega=\int_{\Omega_{s}} w \tilde{u}_{i} d \Omega-\frac{\Delta t}{p} \int_{\Omega_{s}} \frac{a p^{n+1}}{\partial x_{i}} d \Omega$,

after application of divergence theorem and eliminating boundary integrals over which the test function vanishes, the following form is obtained

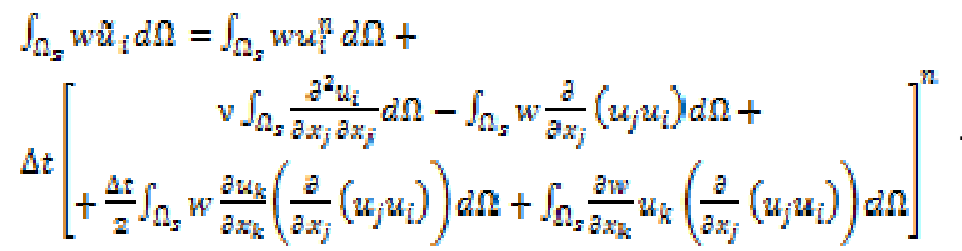

The matrix in (13) and (14) formulated by integration of the full trial functions can be used in either a consistent or lumped form similarly as "mass" matrix in the transitional dynamic analysis. In the article the lumped or diagonal form is used because it simplifies the procedure of matrix inversion. The fractional time step algorithm described in the article can be summarized for each time step every time step (Kovarik et al., 2014) as follows:

- Step 1: Computation of the intermediate velocity field $\tilde{u}$ from the velocities at the previous time step using (14),

- Step 2: Projection of the intermediate velocities to divergence-free plane - solution of pressure equation (9),

- Step 3: Computation of velocities at the current time step from (14).

\section{Numerical Example - flow over backward-facing step}

Based on the theoretical aspects of MLPG and fractional step algorithm, the CFD solver was developed using $\mathrm{C}_{++}$with OpenBLAS 0.9 (compiled with LAPACK interface). The often used test case for the CFD laminar incompressible flow solver is the flow over a backward-facing step. The geometrical configuration and imposed boundary conditions used here are similar to those used by Erturk (2008) or Najafi et al. (2012) (see Fig. 2). The inlet velocity is horizontal and represents the fully developed flow with a parabolic shape. The values of the inlet profile velocities are defined using following equation (Kovarik et al., 2014)

$u=24(y-0.5)(1-y)$.

The maximum inflow velocity is $u_{\max }=1.5$, and the average inflow velocity is $u_{a}=1$. The Reynolds number for this case is defined as

$\operatorname{Re}=\frac{\mathrm{u}_{\mathrm{z}} \mathrm{H}}{v}$.

The height of the flow channel is $H=1$ (see Fig. 2). 


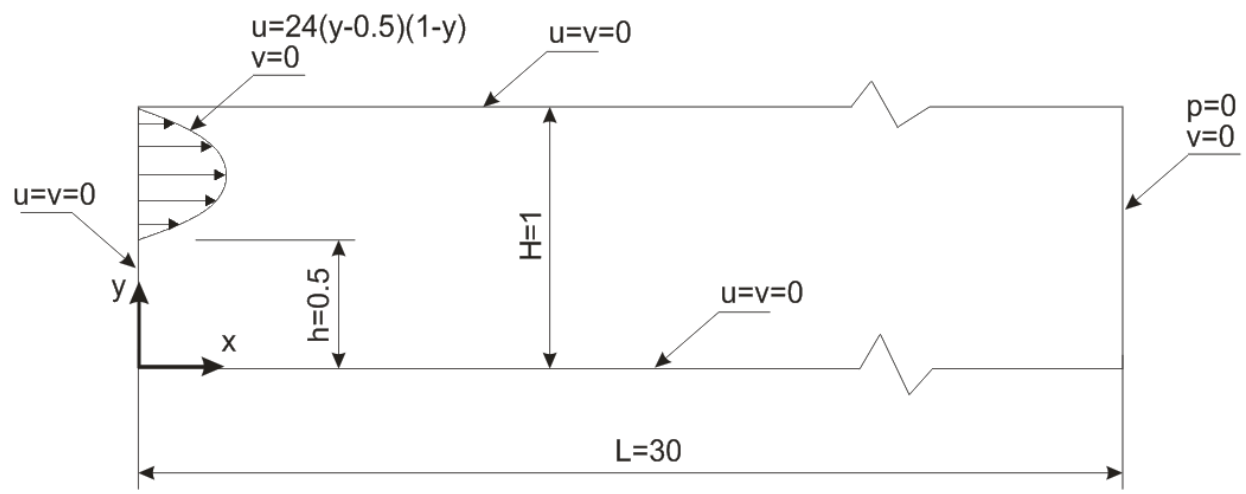

Fig. 2: Backward-facing step, geometry and boundary conditions.

The computational problem domain is represented by uniform nodal distribution that consists of 301 points in the direction of the $x$-axis and 21 in the $y$-axis direction for $0 \leq x \leq 30$. The initial state for the velocity and pressure fields is set to zero.

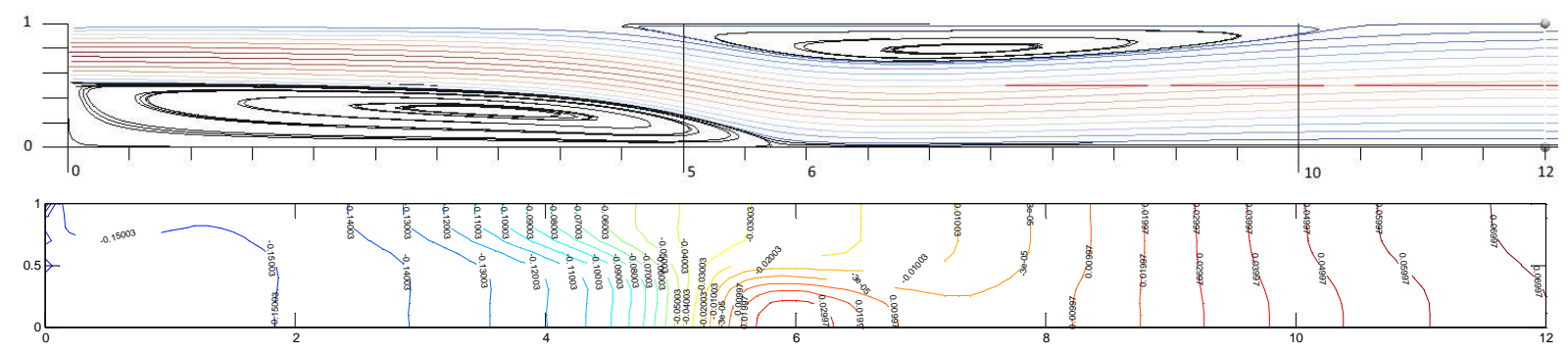

Fig. 3: Streamlines and pressure contours for $\mathrm{Re}=800$.

The statistically steady state solution is reached when the tolerance between two consecutive time steps (13) is lower than a tolerance $\varepsilon$. Fig. 3 represents streamlines and pressure contours for $R e=800$ and shows a good agreement in the case of primary reattachment length (Kovarik et al., 2014), and Fig. 4 shows comparison of the horizontal velocity profiles in two vertical cross-sections $(x=3$ and $x=7)$, with values computed by Erturk (2008).
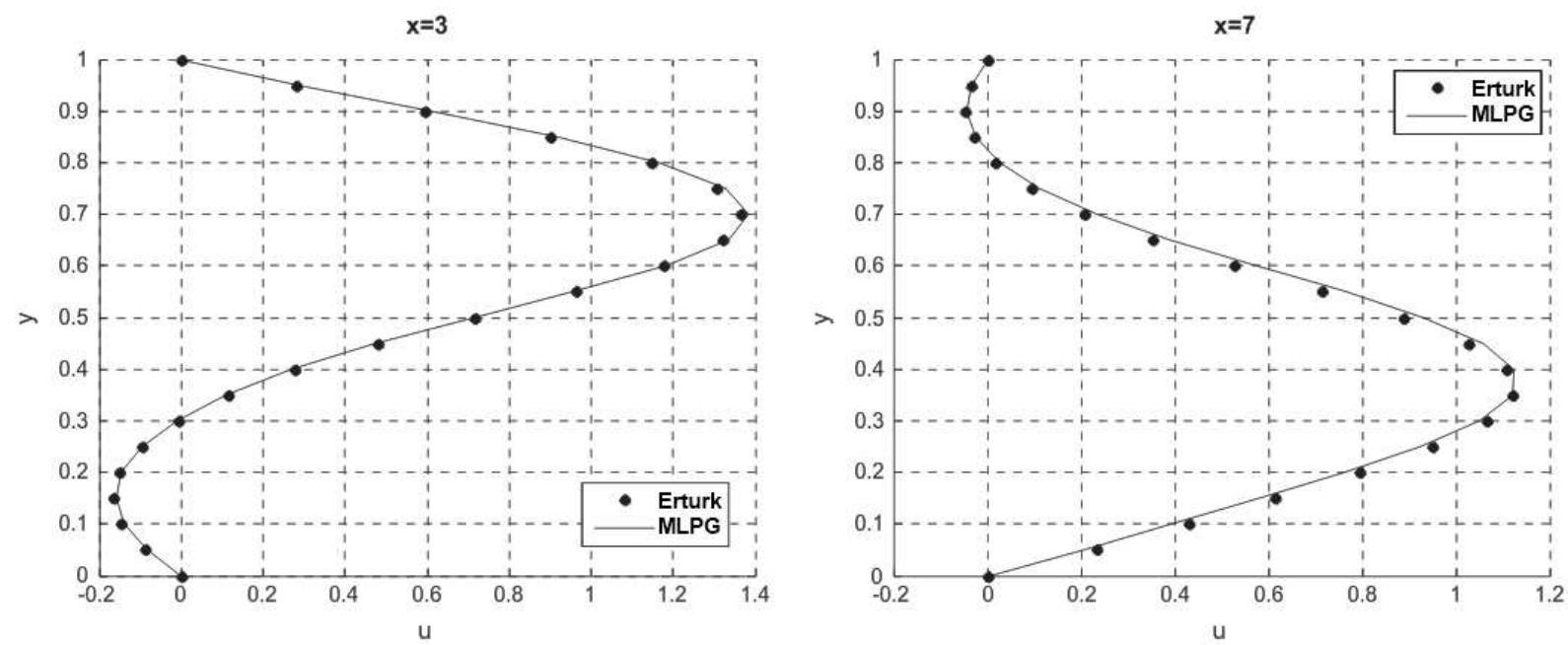

Fig. 4: Comparison of the horizontal velocities in profiles $x=3$ and $x=7$ for $R e=800$.

The Figure 4 shows that the results computed for the low Reynolds numbers are in very good agreement with the other results presented by Erturk (2008) and Kovarik et al. (2014). The results obtained for higher Reynolds number values $(R e>800)$ shows only moderate agreement with the experimental results of Armaly et al. (1983). The reason for such a difference in the computational 
results and experimental data is that for higher $R e$ the flow becomes three dimensional and it's impossible to render all flow aspects using the 2D numerical simulation.

\section{Conclusions}

In the article the numerical simulation of the incompressible laminar viscous flow over backward-facing step is presented. The meshless local Petrov-Galerkin (MLPG) method is adopted for the solution of incompressible Navier-Stokes equations expressed using primitive variables. Treatment of the nonlinear convection term and the Babuska-Brezzi condition is realized using the fractional step algorithm. The ability and accuracy of the two dimensional MLPG fluid dynamics solver was presented by solving backward-facing step flow problem with reasonable accuracy when compared to solutions obtained by other authors and experimental data.

\section{References}

[1] ARMALY, B.F. - DURSTS, F. - PEREIRA, J.C.F. - SCHONUNG, B.: Experimental and theoretical investigation of backward-facing step flow. In: Journal Fluid Mechanics. 1983, Vol. 127, p. 473 96.

[2] ERTURK, E.: Numerical solutions of 2-D steady incompressible flow over a backward-facing step, Part I: high Reynolds number solutions. In: Computational Fluids. 2008, Vol. 37, p. 633 - 55.

[3] IZVOLTOVA, J. - VILLIM, A.: Identification of observations errors by Gauss-Jacobi algorithm. In: Civil and environmental engineering - scientific technical journal. 2012, Vol. 8, Issue 1, p. 13 - 17.

[4] KOVARIK, K.: A meshless solution of two dimensional density-driven groundwater flow. In: Boundary elements and other mesh reduction methods XXXIII. - Southampton, 2011, WIT Press, p. $253-264$.

[5] KOVARIK, K. - MUZIK, J. - MAHMOOD, M.S.: A meshless solution of two-dimensional unsteady flow. In: Engineering Analysis with Boundary Elements. 2012, Vol. 36, p. 738 - 743.

[6] KOVARIK, K. - MUZIK, J. - SITANYIOVA, D.: A fractional step local boundary integral element method for unsteady two-dimensional incompressible flow. In: Engineering Analysis with Boundary Elements. 2014, Vol. 44, p. 55 - 63.

[7] LIN, H. - ATLURI, S.N.: The Meshless Local Petrov-Galerkin Method for Solving Incompressible Navier-Stokes Equations. In: Computer Modeling in Engineering \& Sciences. 2001, p. 117 - 142.

[8] NAJAFI, M. - AREFMANESH, A. - EJILELA, V.: Meshless local Petrov-Galerkin method - higher Reynolds numbers fluid flow applications. In: Engineering Analysis with Boundary Elements. 2012, Vol. 36, p.71 - 85.

[9] SATAPRAHMA, C. - LUADSONGA, A.: The Meshless Local Petrov-Galerkin method for simulating unsteady incompressible fluid flow. In: Journal of the Egyptian Mathematical Society. 2013. 